\title{
Simulation of impulse response for indoor visible light communications using 3D CAD models
}

\author{
Silvestre Pérez Rodríguez ${ }^{*}$, Rafael Pérez Jiménez ${ }^{2}$, Beatriz Rodríguez Mendoza', \\ Francisco José López Hernández $z^{3}$ and Alejandro José Ayala Alfonso ${ }^{1}$
}

\begin{abstract}
In this article, a tool for simulating the channel impulse response for indoor visible light communications using 3D computer-aided design (CAD) models is presented. The simulation tool is based on a previous Monte Carlo ray-tracing algorithm for indoor infrared channel estimation, but including wavelength response evaluation. The 3D scene, or the simulation environment, can be defined using any CAD software in which the user specifies, in addition to the setting geometry, the reflection characteristics of the surface materials as well as the structures of the emitters and receivers involved in the simulation. Also, in an effort to improve the computational efficiency, two optimizations are proposed. The first one consists of dividing the setting into cubic regions of equal size, which offers a calculation improvement of approximately 50\% compared to not dividing the 3D scene into sub-regions. The second one involves the parallelization of the simulation algorithm, which provides a computational speed-up proportional to the number of processors used.
\end{abstract}

Keywords: Visible light communications, Ray-tracing, Impulse response, CAD models, Parallelization

\section{Introduction}

Recently, there has been a growing interest in visible light communications (VLC) in some indoor application scenarios, video/audio transmission for in-home applications, secure network access, or sensor networking [1-8]. Furthermore, wireless optical communications present certain advantages over radiofrequency (RF) transmission that make them suitable in certain specific scenarios. Optical systems do not interfere with RF systems, thus avoiding electromagnetic compatibility restrictions. Moreover, there are no current legal restrictions involving bandwidth allocation and, since radiation is confined by walls, they produce intrinsically cellular networks, which are more secure against deliberate attempts to gain unauthorized access than those relying on radio systems. In this sense, the characterization of indoor VLC channels, their time dispersion, and wavelength response is essential to studying and analyzing the limits in terms of the design and performance offered by such links.

\footnotetext{
*Correspondence: srdguezp@ull.es

'Departamento de Física Fundamental y Experimental, Electrónica y Sistemas, Universidad de La Laguna, 38203, La Laguna, Tenerife, Spain Full list of author information is available at the end of the article
}

Simulating an indoor VLC channel can significantly benefit the design of high performance systems, but requires computationally efficient algorithms and models that accurately fit the characteristics of the channel elements. In order to evaluate the impulse response for indoor VLC channels, two simulation algorithms can be adapted: the Barry and the López-Hernández algorithms. While the Barry algorithm is deterministic and based on an iterative method [9], the López-Hernández algorithm (called the Monte Carlo ray-tracing algorithm) is based on ray-tracing techniques and Monte Carlo method [10], which exhibits a lower computational cost than the Barry algorithm, especially when a high temporal resolution, complex geometries, and a large number of reflections are considered. For this reason, in this article a tool for simulating the impulse response of indoor VLC channels using 3D computer-aided design (CAD) models is presented. The simulation tool is based on the Monte Carlo ray-tracing algorithm [10-12], and allows us to study the VLC signal propagation inside any simulation environment or 3D scene, regardless of its geometric shape, size (area), number of obstacles, etc. The tool features two fully differentiated parts. The first is charged with defining the $3 \mathrm{D}$ scene or the simulation 
environment, which the user can describe by means of any CAD software that is capable of generating or storing the scene in 3DS format. The geometry of the setting where the communications are being established, along with the different material types, emitters, and receivers that comprise the link or links involved in the simulation is specified in the 3D scene. The second element consists of implementing the propagation model. This refers to the mathematical models that characterize the effect of each of the elements present in the simulation environment (reflecting surfaces, emitters, and receivers), and to the simulation algorithm that, aided by these models, allows the channel response to be computed. The part of the tool that implements the propagation model and into which the 3D scene is input is programmed in $\mathrm{C}++$. In addition, so as to improve the computational efficiency of the simulation tool, two optimizations are proposed. The first one consists of dividing the simulation environment into sub-cubes of equal size, so that when a ray is traced in these sub-regions, only those object faces or surfaces that are in the ray propagation path need to be considered. This first optimization allows us to reduce the execution time by approximately $50 \%$ compared to not dividing the 3D scene into sub-regions. The second one consists of parallelizing the simulation algorithm. For each wavelength, the parallelization method proposed involves the equal and static distribution of the rays for computation by different processors, i.e., following a uniform distribution. This optimization results in a calculation speed-up that is essentially proportional to the number of processors used, i.e., when $2,4,8$, and 16 processors are used, the computational speed-up increased by $2,4,8$, and 16 times, respectively, with respect to using a single processor.

This article is organized as follows. In Section 2, the signal propagation model in an indoor VLC channel is defined; i.e., the Monte Carlo ray-tracing algorithm and mathematical models used to characterize the elements of the visible light link are described. Section 3 describes the main features of the simulation tool and its computational complexity, which is compared with an alternate algorithm. The results are discussed in Section 4. Thus, several simulation results are reported to show the potential of the simulation tool and the effects on the computational speed-up due to both optimizations. Finally, Section 5 outlines the conclusions of this article.

\section{Propagation model}

As in conventional infrared wireless communication systems, VLC uses intensity modulation and direct detection for data transmission and detection. In general, for diffuse links, the indoor VLC system consists of an emitter, a receiver, and reflection surfaces. The propagation model is composed by the simulation algorithm and mathematical models used to describe the features of the elements of the optical link. To evaluate the impulse response of the VLC channel, a Monte Carlo ray-tracing algorithm has been adapted [10-12]. In general, the multi-wavelength impulse response for an arbitrary position of emitter $E$ and receiver $R$ can be expressed as an infinite sum of the form [9]

$$
\begin{aligned}
h(t ; E, R, \lambda)= & h^{(0)}(t ; E, R, \lambda) \\
& +\sum_{k=1}^{\infty} h^{(k)}(t ; E, R, \lambda)
\end{aligned}
$$

where $h^{(0)}(t: E, R, \lambda)$ represents the line-of-sight (LOS) impulse response, $h^{(k)}(t ; E, R, \lambda)$ is the impulse response of the light undergoing $k$ reflections, i.e., the multiple-bounce impulse responses, $\lambda$ is the wavelength, and $t$ is the time.

\subsection{LOS impulse response}

Given an emitter $E$ and a receiver $R$ in an environment free of reflectors (see Figure 1), with a large distance $d_{0, R}$ between both [9], the LOS impulse response is approximately

$$
h^{(0)}(t ; E, R, \lambda)=\frac{1}{\left(d_{0, R}\right)^{2}} R_{E}(\varphi, n, \lambda) A_{\mathrm{eff}}(\psi) \delta\left(t-\frac{d_{0, R}}{c}\right)
$$

where $R_{E}(\varphi, n, \lambda)$ represents the generalized Lambertian model used to approximate the radiation pattern of the emitter, $c$ is the speed of light and $A_{\text {eff }}(\psi)$ is the effective signal-collection area of the receiver [9], which is given by

$$
A_{\text {eff }}(\psi)=A_{R} \cos (\psi) \operatorname{rect}\left(\frac{\psi}{\mathrm{FOV}}\right)
$$

where $\operatorname{rect}(x)$ is the rectangular function, whose value is 1 for $|x| \leq 1$ and 0 for $|x|>1, A_{R}$ the physical area of the

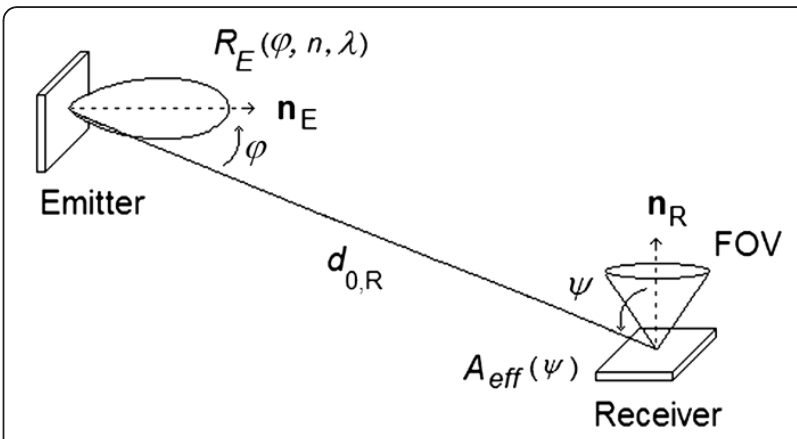

Figure 1 Emitter and receiver geometry without reflectors. 
receiver, and FOV its field of view (semi-angle from the surface normal). In general, the emitter is modeled using a generalized Lambertian radiation pattern for each wavelength, and has axial symmetry (independent of $\gamma$ )

$$
\begin{aligned}
R_{E}(\varphi, n, \lambda)= & \frac{n+1}{2 \pi} P_{E}(\lambda) \cos ^{n}(\varphi), \\
& -\pi / 2 \leq \varphi \leq \pi / 2, \quad 0 \leq \gamma \leq 2 \pi
\end{aligned}
$$

where $n$ is the mode number of the radiation lobe, which specifies the directionality of the emitter [9]. Integrating $P_{E}(\lambda)$ over the emitter wavelength interval yields the nominal power $P_{E}$ emitted by the emitter.

\subsection{Multiple-bounce impulse responses}

Consider an emitter and a receiver in an environment with reflectors. Radiation from the emitter can reach the receiver after any number of reflections (see Figure 2). In order to calculate the multiplebounce impulse responses using the Monte Carlo ray-tracing algorithm, many rays are generated at the emitter position with a probability distribution equal to its radiation pattern $R_{E}(\varphi, n, \lambda)$. The power of each ray generated is initially $P_{E}(\lambda) / N(\lambda)$, where $N=N(\lambda)$ is the number of rays used to discretize the source for each wavelength. When a ray impinges on a surface, the reflection point is converted into a new optical source, thus a new ray is generated with a probability distribution provided by the reflection pattern of that surface. The process continues throughout the maximum simulation time, $t_{\max }$. After each reflection, the power of the ray is reduced by the reflection coefficient of the surface $\rho(\lambda)$ and the reflected power reaching the receiver is computed.
For each wavelength, the power contribution of the $i$ th ray generated by emitter $(1 \leq i \leq N)$ after $k$ reflections can be expressed by

$$
\begin{aligned}
P_{i, k}(E, R, \lambda) & =\frac{1}{\left(d_{k, R}\right)^{2}} R_{S}\left(\theta_{k, R}, \theta^{\prime}, \lambda\right) A_{\mathrm{eff}}\left(\psi_{k, R}\right) \\
t_{i, k} & =\left(\sum_{j=1}^{k} \frac{d_{j-1, j}}{c}\right)+\frac{d_{k, R}}{c}
\end{aligned}
$$

where $t_{i, k}$ represents the time instant in which the power is detected by the receiver and $R_{S}\left(\theta_{k, R}, \theta, \lambda\right)$ is the model used to describe the reflection pattern. In this article, Phong's model has been used [11,13]. In contrast to Lambert's model, this model is able to approximate reflections consisting of both specular and diffusive components, which are described by

$$
\begin{aligned}
R_{S}\left(\theta_{k, R}, \theta^{\prime}, \lambda\right)= & \rho_{k}(\lambda) P_{\text {inc }}(\lambda)\left[\frac{r_{d}(\lambda)}{\pi} \cos \left(\theta_{k, R}\right)\right. \\
& \left.+\left[1-r_{d}(\lambda)\right] \frac{m(\lambda)+1}{2 \pi} \cos ^{m}\left(\theta_{k, R}-\theta^{\prime}\right)\right]
\end{aligned}
$$

The surfaces in Phong's model are defined by three parameters for each wavelength: the reflection coefficient $\rho_{k}(\lambda)$, the percentage of incident signal that is reflected diffusely $r_{d}(\lambda)$, and the directivity of the specular component of the reflection $m(\lambda)$. The parameters $r_{d}(\lambda)$ and $m(\lambda)$ can be considered as independent for each wavelength (unless in these simulations we consider them as constant). Furthermore, $\theta_{k, R}$ and $\theta^{\prime}$ are the observation angle and the incidence angle, respectively. Lastly, $P_{\text {inc }}(\lambda)$ represents the optical power of the

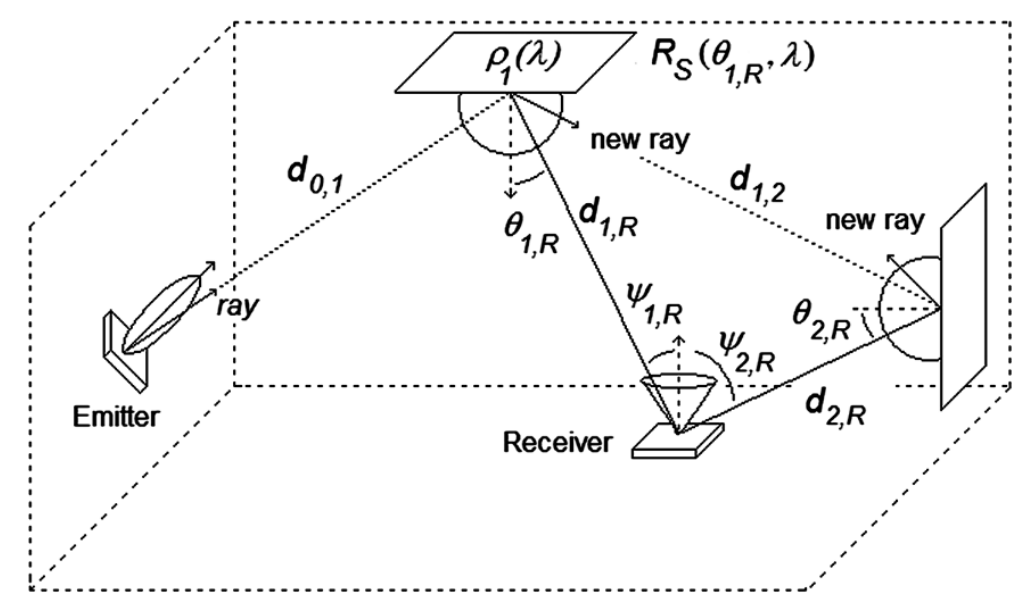

Figure 2 Emitter and receiver geometry with reflectors. The dashed lines represent the ray paths, and the solid ones the power contributions. 
incident ray before undergoing the $k$ th reflection, which is given by

$$
P_{\text {inc }}(\lambda)=\frac{P_{E}(\lambda)}{N} \prod_{j=1}^{k-1} \rho_{j}(\lambda)
$$

Summing the power contributions $P_{i, k}$ in (5) for the total number $N$ of rays, each undergoing a maximum of $K$ reflections, and using the Dirac delta function to symbolize the time instants $t_{i, k}$, yields the multiplebounce response, which is described by

$$
\begin{aligned}
\sum_{k=1}^{\infty} h^{(k)}(t ; E, R, \lambda)= & \sum_{i=1}^{N} \sum_{k=1}^{K} P_{i, k}(E, R, \lambda) \cdot \delta\left(t-t_{i, k}\right) \\
= & \sum_{i=1}^{N} \sum_{k=1}^{K} \frac{1}{\left(d_{k, R}\right)^{2}} R_{S}\left(\theta_{k, R}, \theta^{\prime}, \lambda\right) A_{\mathrm{eff}}\left(\psi_{k, R}\right) \\
& \times \cdot \delta\left(t-\left(\sum_{j=1}^{k} \frac{d_{j-1, j}}{c}\right)-\frac{d_{k, R}}{c}\right)
\end{aligned}
$$

Substituting Equations (2) and (8) in (1), the total impulse response as a function of wavelength can be expressed as

$$
\begin{aligned}
h(t ; E, R, \lambda)= & \frac{1}{\left(d_{0, R}\right)^{2}} R_{E}(\varphi, n, \lambda) A_{\mathrm{eff}}(\psi) \delta\left(t-\frac{d_{0, R}}{c}\right) \\
& +\sum_{i=1}^{N} \sum_{k=1}^{K} \frac{1}{\left(d_{k, R}\right)^{2}} R_{S}\left(\theta_{k, R}, \theta^{\prime}, \lambda\right) A_{\mathrm{eff}}\left(\psi_{k, R}\right) \\
& \times \cdot \delta\left(t-\left(\sum_{j=1}^{k} \frac{d_{j-1, j}}{c}\right)-\frac{d_{k, R}}{c}\right)
\end{aligned}
$$

Defining $M=t_{\mathrm{max}} / \Delta t$, and assuming as the time origin the arrival of the LOS component, we can express the impulse response histogram as

$$
\begin{aligned}
h(t ; E, R, \lambda)= & \frac{1}{\left(d_{0, R}\right)^{2}} R_{E}(\varphi, n, \lambda) A_{\mathrm{eff}}(\psi) \delta(t) \\
& +\sum_{\substack{n-1 \\
M-1}}^{N_{n}} \sum_{k=1}^{K_{n}} \frac{1}{\left(d_{k, R}\right)^{2}} R_{S}\left(\theta_{k, R}, \theta^{\prime}, \lambda\right) A_{\mathrm{eff}}\left(\psi_{k, R}\right) \\
& \times \delta(t-n \Delta t)
\end{aligned}
$$

where $n$ symbolizes the $n$th interval time (width $\Delta t$ ) or bin of the power histogram. Furthermore, $K_{n}$ and $N_{n}$ are the number of reflections of the $i$ th ray and the number of rays that contribute in the $n$th time interval, respectively. This equation can also be written as

$$
\begin{aligned}
h(t ; E, R, \lambda)= & \frac{1}{\left(d_{0, R}\right)^{2}} R_{E}(\varphi, n, \lambda) A_{\mathrm{eff}}(\psi) \delta(t) \\
& +\sum_{n=1}^{M-1} P_{n}(E, R, \lambda) \cdot \delta(t-n \Delta t)
\end{aligned}
$$

where $P_{n}$ represents the total received power in the $n$th time interval. $P_{n}$ is calculated as the sum of the power of the $N_{n}$ rays that contribute in that interval, which is given by

$$
\begin{aligned}
P_{n}(E, R, \lambda) & =\sum_{i=1}^{N_{n}} P_{i, n}(E, R, \lambda) \\
& =\sum_{i=1}^{N_{n}} \sum_{k=1}^{K_{n}} \frac{1}{\left(d_{k, R}\right)^{2}} R_{S}\left(\theta_{k, R}, \theta^{\prime}, \lambda\right) A_{\text {eff }}\left(\psi_{k, R}\right)
\end{aligned}
$$

where $P_{i, n}$ is the total reflected power reaching the receiver in the $n$th time interval due to the $i$ th ray propagation.

\subsection{Error estimate of the simulated impulse responses}

The use of an algorithm based on the Monte Carlo method allows for the error in computing the impulse response to be estimated with just one simulation run, as long as the number of rays is large enough. Although different error estimates are obtained for several simulations, we can be confident that the standard deviation of the estimates decreases as the number of rays is increased. Moreover, the method allows for the accuracy of the results to be assessed. The partial results of one simulation can also be used to achieve a more accurate solution by selecting a suitable number of rays.

In previous research $[14,15]$, the equation that provides an error determination when computing the impulse response was reported, which can be estimated as the square root of the total received power variance, $\operatorname{var}\left(P_{n}(\lambda)\right.$ ), in the $n$th time interval (width $\Delta t$ ). Therefore, for each wavelength the absolute error is given by

$$
\begin{aligned}
\operatorname{err}\left(P_{n}(\lambda)\right) & =\sqrt{\operatorname{var}\left(P_{n}(\lambda)\right)} \\
& =\sqrt{\sum_{i=1}^{N_{n}} P_{i, n}^{2}(\lambda)-\frac{1}{N}\left(\sum_{i=1}^{N_{n}} P_{i, n}(\lambda)\right)^{2}}
\end{aligned}
$$

where $P_{i, n}$ is the reflected power reaching the receiver ( $i$ th ray, $n$th time interval), $N_{n}$ is the number of rays that contribute in that interval, $N$ is the number of rays used to discretize the source, and $P_{n}$ is the total received power in the $n$th time interval, which was described in (12). Therefore, the relative error in a time interval $\Delta t$ can be expressed as

$$
\operatorname{rel} \operatorname{err}\left(P_{n}(\lambda)\right)=\sqrt{\frac{\sum_{i=1}^{N_{n}} P_{i, n}^{2}(\lambda)}{\left(\sum_{i=1}^{N_{n}} P_{i, n}(\lambda)\right)^{2}}-\frac{1}{N}}
$$

The Monte Carlo method establishes that the error is proportional to $1 / \sqrt{N}$, i.e., once we have computed the error using $N$ rays, we can estimate what error would result from launching more rays. This is important 
because we can estimate the number of rays needed to obtain results with the accuracy appropriate to the matter of interest. For example, if the relative error obtained is $4.5 \%$ for 100,000 rays, the number of rays needed to decrease the error to $2 \%$ is $500,000(2 \%=4.5 \% \sqrt{100,000} /$ $\sqrt{500,000})$. Considering $\Delta T$ as the time elapsed between the initial time and every subsequent simulation instant, Equation (14) allows us to determine the cumulative error along the simulation time. Therefore, the relative cumulative error in a time interval $\Delta T$ can be described by

$$
\text { rel cum } \operatorname{err}\left(P_{n}(\lambda)\right)=\sqrt{\frac{\sum_{i=1}^{N_{\Delta T}} P_{i, n}^{2}(\lambda)}{\left(\sum_{i=1}^{N_{\Delta T}} P_{i, n}(\lambda)\right)^{2}}-\frac{1}{N_{f}}}
$$

where $N_{\Delta T}$ is the number of rays that contribute in $\Delta T$ and $N_{f}$ is the number of flights of rays along that interval time.

\section{Features of the simulation tool}

In the following sections, the elements that constitute the simulation tool are described. Moreover, an equation that describes the computational complexity of the simulation algorithm based on the number of rays, number of reflections, and geometric complexity of the simulation environment is presented.

\subsection{Description of the simulation tool}

The simulation tool developed allows us to estimate the impulse response of indoor VLC channels in time and wavelength using 3D CAD models. Figure 3 shows the block diagram of the simulation tool. The diagram reveals the two key elements that comprise the simulation software: the inputs of the simulation tool that the user specifies by means of various files, and the propagation model described in Section 2, which consists of the simulation algorithm and the mathematical models that characterize the effect of each element present in the optical link. In addition, the tool also includes a utility for displaying and analyzing the program's execution trace through a $3 \mathrm{D}$ viewer developed using Java $3 \mathrm{D}$. The inputs of the simulation tool consist of the geometry of the simulation environment or 3D scene, the parameters of the reflection pattern of the materials comprising the reflective surfaces, the emitter and receiver locations, and other simulation parameters such as the number of rays, the maximum number of reflections, the maximum simulation time, emitter and receiver orientations, the emitter's modal index, the receiver's field of view, etc. While the user can describe the simulation environment geometry using any CAD software that is capable of generating or storing the $3 \mathrm{D}$ scene in a 3DS file, the remaining inputs are specified by means of auxiliary text files.

One of the main features of this tool is that it allows us to study the VLC signal propagation inside any simulation environment, regardless of its geometric shape, size (area), number of obstacles in its interior, etc. In general, any CAD software capable of generating 3D vector-type graphics and storing them in a $3 \mathrm{DS}$-format file can be used. The 3DS file format, currently one of the most complete and widely used, contains information on meshes, material attributes, bitmap references, textures, display configurations, camera positions, luminosity, and even data on object animations. The meshes comprise the elements or objects in the 3D scene, and consist of groups of triangles or faces. Each of these faces is defined by three vertices and has associated with it the properties of the material of which it is made, such as visibility, etc. These properties allow us to establish the reflective characteristics of the materials present in the simulation environment, that is, the way in which the incident rays are reflected. The simulation tool was developed in the $\mathrm{C}++$ programming language and a lib3ds programming library was used to make it easier to work with the 3DS format.

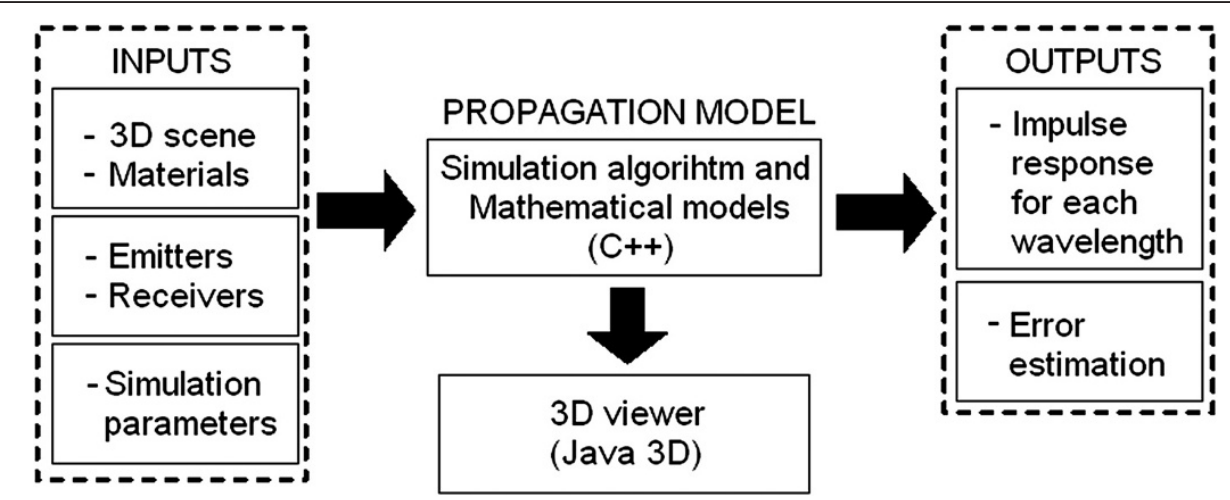

Figure 3 Block diagram of simulation tool. 


\subsection{Computational complexity}

In contrast to other methods [8,9], the Monte Carlo raytracing algorithm allows for the evaluation of the impulse response for environments with complex geometries with no meaningful increase in computational cost, especially when a high temporal resolution, and a large number of reflections are considered. This can be explained by the number of elementary calculations that is performed: $k N$ $N_{F}$ where $N$ is the number of rays for each wavelength, $k$ is the number of reflections that are considered, and $N_{F}$ is the number of faces or triangles that define the geometry. An elementary calculation is defined as the calculation of power contribution and delay from a point source (emitter or reflection point of a ray) to the receiver, as in (5), and the assessment of the propagation of the new generated ray to determine a new point source. This computational cost can be compared with other deterministic algorithms, such as Barry's algorithm [9], where a total of $\left(N_{C}\right)^{k}$ elementary calculations is performed, and $N_{C}$ is the number of elements into which the reflecting surfaces are divided. Therefore, the Monte Carlo ray-tracing algorithm requires a smaller amount of computational effort for a large number of reflections $(k)$ and a large number of triangles or faces $\left(N_{F}\right)$. We can also observe that the area of the triangle is not important, in contrast to Barry's algorithm, where the number of reflecting elements $N_{C}$ depends upon the size of the surfaces. Moreover, Monte Carlo raytracing algorithms allow for the evaluation of the confidence levels of the simulation results. Despite being very accurate, deterministic methods do not allow for an easy evaluation of the error due to discretization. Also, they can compute the impulse response for just the lowerorder reflections, allowing the simulation to be conducted in a reasonable amount of time since the run time is exponential in $k$. Thus, for example, to compute the $k=3$ bounce impulse response with $N_{C}=2,776$ elements (an empty rectangular room of $7.5 \times 5.5 \times 3.5 \mathrm{~m}^{3}$ defined by six surfaces, $N_{F}=12$ triangles), the number of elementary calculations is roughly $2.1 \times 10^{10}\left(5.9 \times 10^{13}\right.$ for $\left.k=4\right)$. The ray-tracing algorithm is able to obtain a simulated impulse response for the same room with a relative error of less than $1 \%$ using $N=10,000,000$ rays, which is equivalent to $3.6 \times 10^{8}$ elementary calculations $\left(4.8 \times 10^{8}\right.$ for $\left.k=4\right)$. In short, for $k=3$ reflections, the Monte Carlo ray-tracing algorithm improves the computational efficiency 58-fold in comparison to using Barry's algorithm. For $k=4$ reflections, the improvement is $1.23 \times 10^{5}$ fold.

\section{Results}

In this section, we present several simulation results to show the potentiality of the simulation tool to approximately characterize the impulse response of indoor VLC channels. Moreover, the effects on the computational speed-up due to the two optimizations proposed for improving the computational efficiency are discussed.

\subsection{Application example}

As an example of an application of the simulation tool developed, we studied the propagation of visible light in the simulation setting depicted in Figures 4 and 5 . The $3 \mathrm{D}$ scene is a $1.0 \times 1.0 \mathrm{~m}^{2}$ hexagonal structure with different objects or obstacles inside. The emitter and receiver are located on wall 1 and oriented towards the interior of the 3D scene, i.e., there is no LOS communication between emitter and receiver (see Figure 5). We should note that even though this example considers a single emitter and receiver, the tool can be used to simulate the presence of multiple emitters and receivers in any $3 \mathrm{D}$ scene. To model the scene, we used the Blender graphic design program because it offers multi-platform support in a freeware product whose output 3DS file includes the simulation environment geometry, emitter
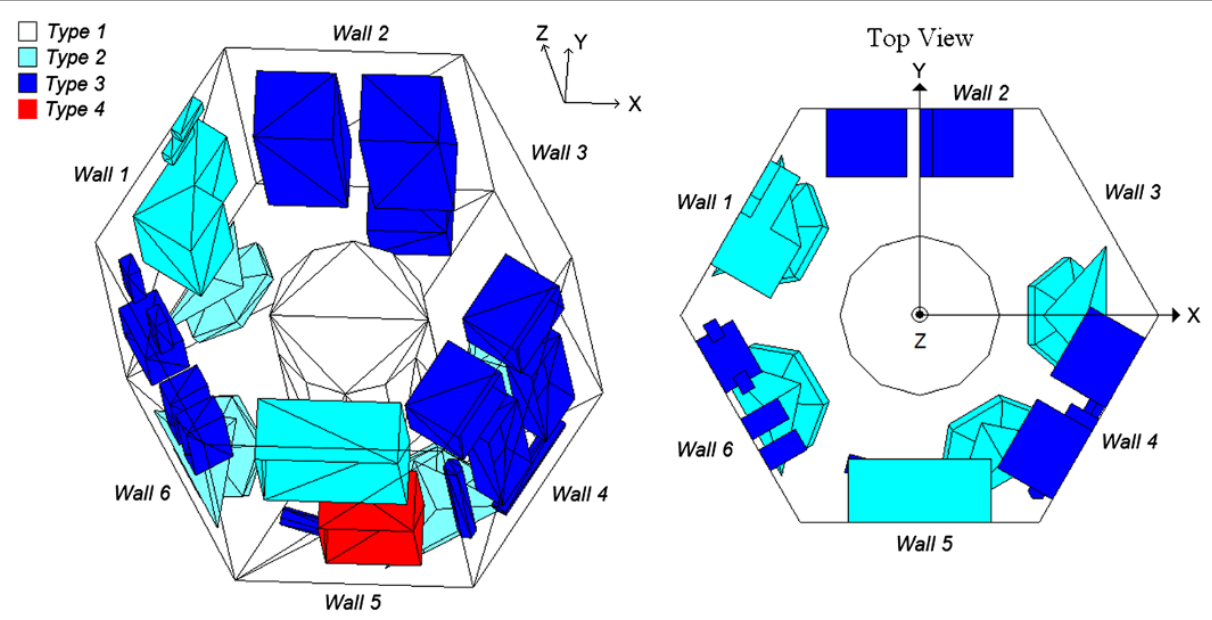

Figure 4 Simulation environment (3D scene). 


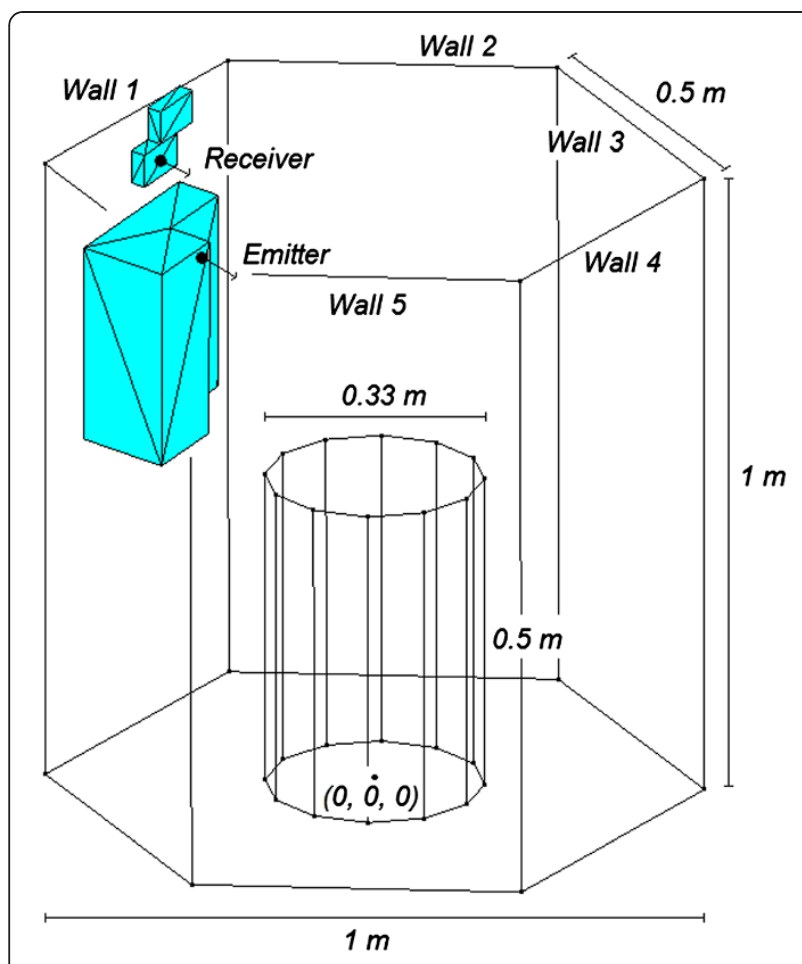

Figure 5 Emitter and receiver locations and 3D scene dimensions.

and receiver locations, as well as the characteristics of the different materials on the triangles that comprise the simulation environment. The 3DS-format file generated by the graphic design software constitutes one of the inputs to the simulation tool, which is capable of

Table 1 Simulation parameters

\begin{tabular}{|c|c|c|c|}
\hline & \multicolumn{2}{|l|}{ Parameter } & Value \\
\hline \multirow[t]{4}{*}{ Emitter } & \multicolumn{2}{|l|}{ Mode $(n)$} & 1 \\
\hline & \multicolumn{2}{|l|}{$\overline{P_{E}}(\lambda), \mathrm{W}$} & $1 / 3$ \\
\hline & \multicolumn{2}{|l|}{ Position $(x, y, z), \mathrm{m}$} & $\overline{(-0.25,0.14,0.83)}$ \\
\hline & \multicolumn{2}{|l|}{ Orientation } & $90^{\circ}, 330^{\circ}$ \\
\hline \multirow[t]{4}{*}{ Receiver } & \multicolumn{2}{|c|}{ Active area $\left(A_{R}\right), \mathrm{cm}^{2}$} & 1 \\
\hline & \multicolumn{2}{|l|}{ Position $(x, y, z), m$} & $\overline{(-0.33,0.24,0.91)}$ \\
\hline & \multicolumn{2}{|l|}{ Orientation } & $90^{\circ}, 330^{\circ}$ \\
\hline & \multicolumn{2}{|l|}{ FOV } & $85^{\circ}$ \\
\hline Resolution & \multicolumn{2}{|l|}{$\Delta t, \mathrm{~ns}$} & 0.2 \\
\hline Bounces & \multicolumn{2}{|l|}{$k$} & 10 \\
\hline Number of rays & \multicolumn{2}{|l|}{$N(\lambda)$} & 500,000 \\
\hline Materials (Type) & $\rho_{\text {Blue }} \rho_{\text {Green }} \rho_{\text {Red }}$ & $\overline{r_{d}(\forall \lambda)}$ & $m(\forall \lambda)$ \\
\hline Wood (1) & 0.250 .430 .73 & 1 & - \\
\hline White marble (2) & 0.700 .770 .82 & 0.5 & 230 \\
\hline Aluminium metal (3) & 0.450 .490 .52 & 0.3 & 250 \\
\hline Black paint (4) & 0.030 .040 .04 & 0 & 138 \\
\hline
\end{tabular}

interpreting the information stored in the graphic file to extract the location of the vertices and triangles used to model the 3D scene. In this case, the scene was modeled using 284 vertices and 416 triangles. Four types of materials with different spectral reflectance characteristics were considered [16]. In addition to the information on the simulation environment provided by the graphic file, other parameters necessary to carry out the simulation must also be specified, such as the number of rays, the maximum number of reflections, the maximum simulation time, emitter and receiver orientations, the emitter's modal index, the receiver's field of view, etc. All of these input parameters are extracted from auxiliary text files. The parameters stored in these files and used in the simulation are shown in Table 1.

In terms of the simulation run time, we note that for a dual-core Intel Xeon $3.20 \mathrm{GHz}$ processor with $1 \mathrm{~GB}$ of RAM running Debian GNU/Linux, the execution time was approximately $21 \mathrm{~min}(1,260 \mathrm{~s})$. Figure 6 illustrates the impulse responses for RGB wavelengths, i.e., $\lambda_{\text {Red }}=$ $635 \mathrm{~nm}, \lambda_{\text {Green }}=525 \mathrm{~nm}$, and $\lambda_{\text {Blue }}=455 \mathrm{~nm}$. The estimates of the relative and relative cumulative errors in computing the impulse responses are also given. We can see that the impulse responses show a similar temporal evolution for each wavelength, though with different power levels. This is because we assumed that only the reflection coefficient of the simulated materials depends on the wavelength, while the remaining parameters are constant (see Table 1). The error curves also present a similar shape. The small differences are due to the random nature of the simulation algorithm. We can see that the relative errors obtained are less than $5 \%$, though in order to ascertain the accuracy of the impulse responses, the relative cumulative error must be examined. The maximum value for the relative cumulative error is given by blue wavelength, which is less than $0.3 \%$.

As regards the computational complexity, since the number of rays considered in the simulation was $N=$ 500,000 , the number of triangles that define the 3D scene was $N_{F}=416$, and the number of reflections was $k$ $=10$, the number of elementary calculations per wavelength is $2.08 \times 10^{9}$. This computational cost can be compared to using Barry's algorithm. If the total area of the reflecting surfaces $\left(7.78 \mathrm{~m}^{2}\right)$ is divided into $N_{C}=778$ elements, i.e., an element area of $100 \mathrm{~cm}^{2}$ is used, the number of elementary calculations is $8.1 \times 10^{28}$. Furthermore, as discussed in Section 3.2, in the Monte Carlo ray-tracing algorithm the size of the reflecting surfaces is not important, in contrast to Barry's algorithm, where the number of reflecting elements $N_{C}$ increases with the size of the surfaces, or total area. Thus, for example, if the area is $70 \mathrm{~m}^{2}$ (a hexagonal structure of $3.0 \times 3.0 \mathrm{~m}^{2}$ ), i.e., $N_{C}=7,000$ elements, the number of operations increases to $2.8 \times 10^{38}$. 


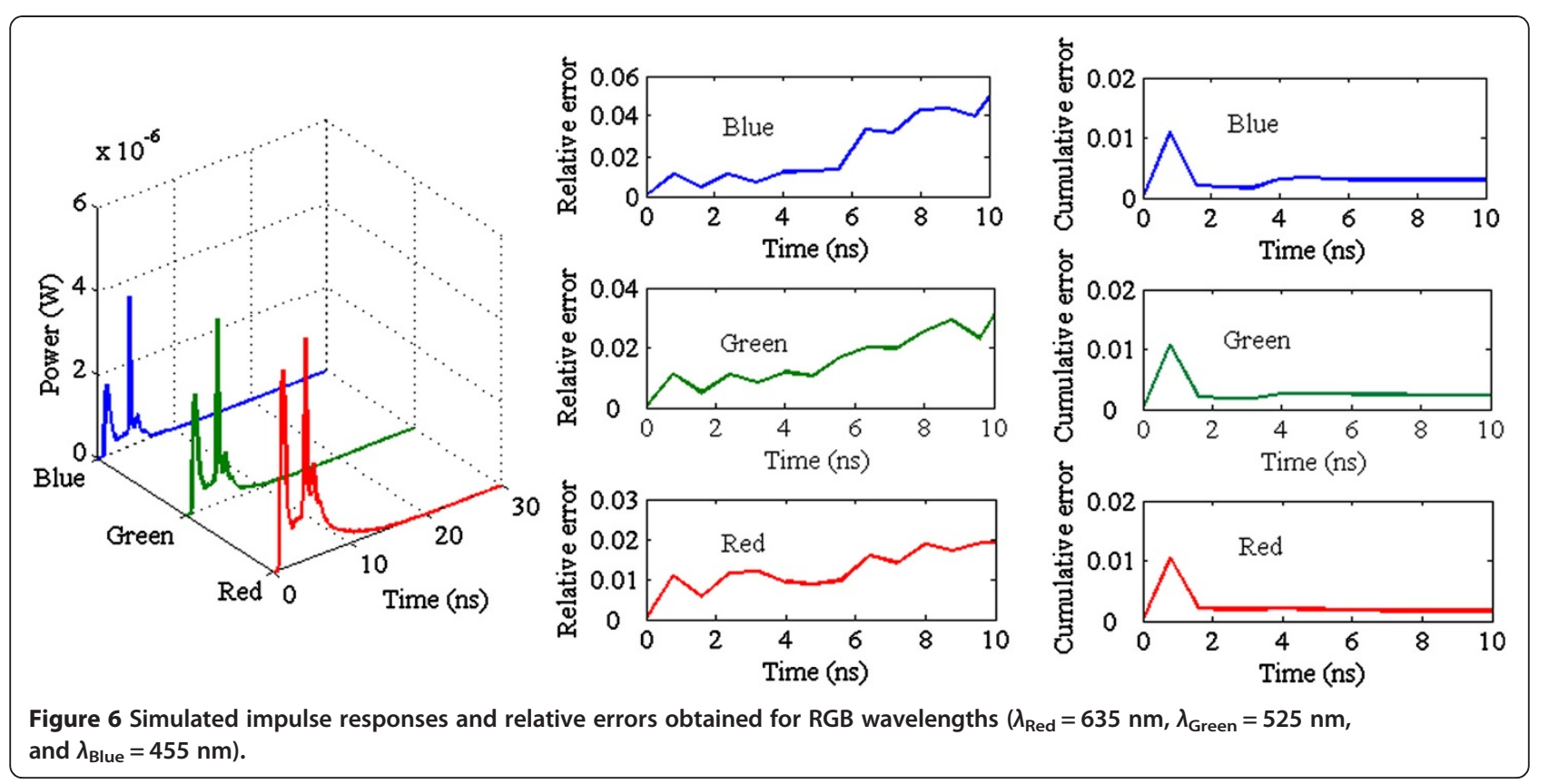

\subsection{Computational speed-up}

In order to improve the computational efficiency of the simulation algorithm, we introduced two optimizations. The first one consists of dividing the 3D simulation environment into a set of cubic sub-regions of equal size. The number of divisions to be made at each edge $c$ can be specified in the tool, thus generating a total of $c^{3}$ grids. When tracing a ray, these grids allow for only those object faces and/or surfaces that are in the propagation path of the ray to be considered. This technique is equivalent to simplifying the ray propagation by considering only those surfaces that are actually involved in the propagation process, and is used for ray-tracing urban field prediction models [17]. With this optimization, the computation time is reduced without affecting the accuracy of the results. The second optimization is the parallelization of the Monte Carlo method. The Monte Carlo method is a numerical statistical method based on the generation of a set of random numbers to compute a set of results associated with each. The final solution is obtained by combining each sub-result. The parallelization of the Monte Carlo method usually involves distributing the computation associated with each random number to the various processors used in the execution. One possibility is to distribute each computation statically, that is, dividing the computation equally among all the processors. If the computational effort associated with each random number varies too much, a static distribution could result in some processors completing their work well before others do. In these cases, methods for balancing the workload are used in an effort to make better use of the resources available. Since workload balancing methods introduce a certain additional load on the processors, these methods should not be used when a static workload distribution will yield a good use of resources. In the case at hand, it is reasonable to assume that a static distribution will yield a better result than a balanced distribution due to the negligible variability in the computational cost associated with each ray; that is, it is unlikely that any one processor will have to compute a large amount of high-cost rays while others compute low-cost rays. Although each ray undergoes a different number of reflections, when the number of rays is large, the average number of reflections experienced by rays assigned to each processor is very similar. Thus, for example, for the simulation environment shown in Figure 4 with 500,000 rays, unlimited reflections, assuming that the minimum power detected by the photodetector is

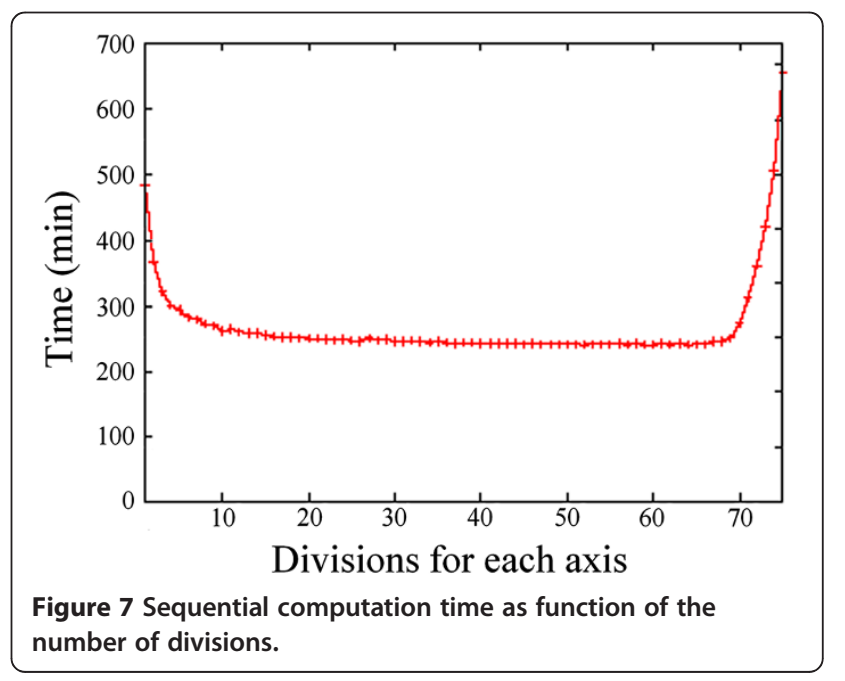




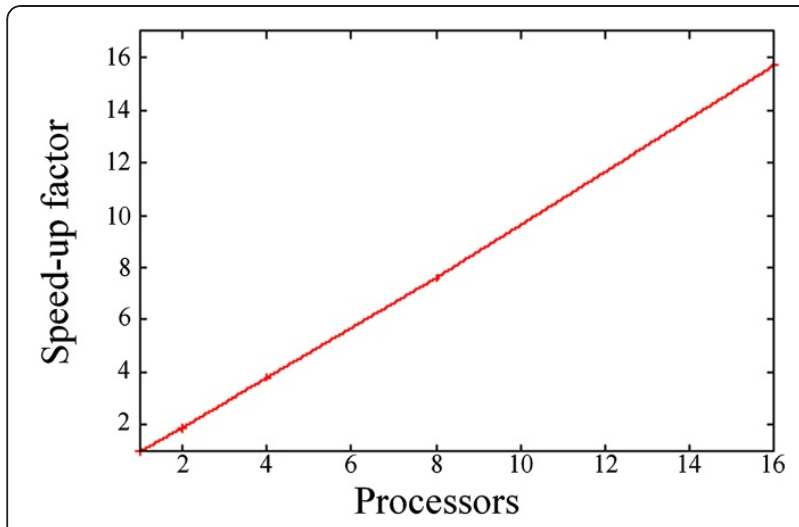

Figure 8 Speed-up factor as function of the number of processors.

$10^{-12} \mathrm{~W}$, and statically distributing the rays among 16 processors, the average number of reflections per processor is $15.01 \pm 0.02$. When a smaller number of processors is used, the number of rays assigned to each processor is greater, resulting in a variation in the average number of reflections per processor of less than 0.02 ( 0.001 for two processors). That is why we propose the utilization of the static ray distribution parallelization method, where the rays are assigned to each processor with equal probability, i.e., following a uniform distribution. Specifically, for each emitter involved in the simulation, each processor will be tasked with simulating a subset of rays for each wavelength.

In order to evaluate the effect of both optimizations on the computational speed-up, several simulations were performed using the same simulation environment and parameters as in the previous section (see Figure 4 and Table 1), though 24 simulated receivers were used, located in different positions within the 3D scene. For this case, without applying the optimizations, the simulation run time was approximately $472 \mathrm{~min}(28,332 \mathrm{~s})$. Figure 7 shows the resulting sequential computing time as a function of the number of divisions, which allow us to determine the computational speed-up due to the optimization involving the use of sub-regions. The experiments were conducted on a Debian GNU/Linux cluster with eight dual-core Intel Zeon $3.20 \mathrm{GHz}$ processors with 1 GB of RAM linked via a Gigabit Ethernet connection. The results clearly show that the use of grids decreases the computation time, though if the number of divisions is increased too much, the simulation performance suffers. This is due primarily to the fact that the initialization time and the memory requirements increase considerably with the number of divisions. Specifically, this optimization provides a $50.6 \%$ improvement when the number of divisions is 70 , which is the number that exhibits the best results in terms of the execution time. Figure 8 shows the computational speed-up obtained from the second optimization proposed, which was to parallelize the algorithm for 2, 4, 8, and 16 processors. As we can see, the behavior shown by the computational speed-up is practically proportional to the number of processors used, thus verifying the initial assumption that a static distribution is sufficient to ensure the proper use of the available resources.

\section{Conclusions}

In this article, we presented the design and implementation of a simulation tool to estimate the impulse response of indoor VLC channels in time and wavelength using 3D CAD models. The indoor VLC channel simulation can significantly benefit the design of high performance VLC systems, but requires computationally efficient algorithms and models that accurately respond to the characteristics of the channel elements. In this sense, the simulation tool allows us to accurately define the simulation environment with 3D CAD models; furthermore, it is based on the Monte Carlo ray-tracing algorithm, which exhibits a lower computational cost than other algorithms, especially when a high temporal resolution, complex geometries, and a large number of reflections are considered. Therefore, one of the main features of the tool is that, for a given number of reflections and temporal resolution, it allows us to study the VLC signal propagation inside any simulation environment, regardless of its geometric shape, size (area), number of obstacles in its interior, etc., with a high computational efficiency. Finally, in order to improve its computational efficiency, two optimizations were introduced. The first consisted of dividing the simulation environment into sub-cubes of equal size so that when a ray is traced in these sub-regions, only those object faces or surfaces that are in the ray propagation path need to be considered. Defining the optimum number of divisions as the maximum possible value that does not saturate the node in terms of the amount of memory required, this first optimization yielded a $50.6 \%$ decrease in execution time compared to not dividing the 3D scene into sub-regions. The second optimization consisted of parallelizing the simulation algorithm based on an equal and static distribution of the rays generated at the emitter among the available processors, i.e., assigning the rays to each processor by means of a uniform distribution. This optimization resulted in a computational speed-up that is essentially proportional to the number of processors used. In short, when $2,4,8$, and 16 processors are used, the speed-up factor increased by $2,4,8$, and 16 times, respectively, with respect to using a single processor.

Competing interests

The authors declare that they have no competing interests.

\section{Acknowledgments}

This study was funded in part by the Spanish Ministerio de Ciencia e Innovación (project TEC2009-14059-C03-02/01/03), Plan E (Spanish Economy and 
Employment Stimulation Plan), and the Government of the Canary Islands (project SolSubC200801000306).

\section{Author details}

'Departamento de Física Fundamental y Experimental, Electrónica y Sistemas, Universidad de La Laguna, 38203, La Laguna, Tenerife, Spain. ${ }_{2}^{2}$ Instituto para el Desarrollo Tecnológico y la Innovación en Comunicaciones - IDeTIC, Universidad de Las Palmas de Gran Canaria, 35017, Las Palmas de Gran Canaria, Spain. ${ }^{3}$ Centro de Domótica Integral - CeDint, Universidad Politécnica de Madrid, 28223, Madrid, Spain.

Received: 31 March 2012 Accepted: 27 November 2012

Published: 12 January 2013

\section{References}

1. KD Langer, J Vucic, C Kottke, LF del Rosal, S Nerreter, J Walewski, Advances and prospects in high-speed information broadcast using phosphorescent white-light LEDs, in Proceeding of 11th International Conference on Transparent Optical Networks (ICTON '09), Azores, Portugal, 28 June-2 July, 2009, pp. 1-6

2. KD Langer, J Grubor, Recent developments in optical wireless communications using infrared and visible light, in Proceeding of 9th International Conference on Transparent Optical Networks (ICTON '07), Rome, Italy, 1-5 July 2007, vol. 3, pp. 146-151

3. T Komine, M Nakagawa, Fundamental analysis for visible-light communication system using LED lights. IEEE Trans. Consum. Electron. 50(1), 100-107 (2004). doi:10.1109/TCE.2004.1277847

4. H Lee, Y Kim, K Sohn, Optical wireless sensor networks based on VLC with PLC-Ethernet interface. WASET 81, 245-248 (2011)

5. PA Haigh, H Le Minh, Z Ghassemlooy, Transmitter distribution for MIMO visible light communication systems, in Proceeding of 12th Annual Post Graduate Symposium on the Convergence of Telecommunications, Networking and Broadcasting (PGNet2011), Liverpool, United Kingdom, 27-28 June 2011, pp. 190-193

6. PA Haigh, TT Son, E Bentley, Z Ghassemlooy, H Le Minh, L Chao, Development of a visible light communications system for optical wireless local area networks, in Proceeding of Computing, Communications and Applications Conference (ComComAp), Hong Kong, China, 11-13 June 2012, pp. 351-355

7. E Cho, JH Choi, C Park, M Kang, S Shin, Z Ghassemlooy, CG Lee, NRZ-OOK signalling with LED dimming for visible light communication link, in Proceeding of 16th European Conference on Networks and Optical Communications (NOC), Newcastle-Upon-Tyne, United Kingdom, 20-22 July 2011, pp. 32-35

8. K Lee, H Park, JR Barry, Indoor channel characteristics for visible light communications. IEEE Commun. Lett. 15, 217-219 (2011). doi:10.1109/ Icomm.2011.010411.101945

9. JR Barry, JM Kahn, EA Lee, DG Messerschmitt, Simulation of multipath impulse response for indoor wireless optical channels. IEEE J. Sel. Areas Commun. 11(3), 367-379 (1993). doi:10.1109/49.219552

10. FJ López-Hernandez, R Pérez-Jiménez, A Santamaría, Ray-tracing algorithms for fast calculation of the channel impulse response on diffuse IR-wireless indoor channels. Opt. Eng. 39(10), 2775-2780 (2000). doi:10.1117/1.1287397

11. S Rodríquez, R Pérez-Jiménez, FJ López-Hernádez, O González, A Ayala, Reflection model for calculation of the impulse response on IR-wireless indoor channels using ray-tracing algorithm. Microw. Opt. Technol. Lett. 32(4), 296-300 (2002). doi:10.1002/mop.10159

12. S Rodríguez, R Pérez Jiménez, O González, J Rabadán, BR Mendoza, Concentrator and lens models for calculating the impulse response on IR-wireless indoor channels using a ray-tracing algorithm. Microw. Opt. Technol. Lett 36(4), 262-267 (2003). doi:10.1002/mop.10738

13. CR Lomba, RT Valadas, AM Oliveira Duarte, Experimental characterisation and modelling of the reflection of infrared signals on indoor surfaces. IEE Proc. Optoelectron. 145(3), 191-197 (1998). doi:10.1049/ip-opt:19982020

14. O González, C Militello, S Rodríquez, R Pérez-Jiménez, A Ayala, Error estimation of the impulse response on diffuse wireless infrared indoor channels using a Monte Carlo ray-tracing algorithm. IEE Proc. Optoelectron. 149(5-6), 222-227 (2002). doi:10.1049/ip-opt:20020545

15. O González, S Rodríguez, R Pérez-Jiménez, BR Mendoza, A Ayala, Error analysis of the simulated impulse response on indoor wireless optical channels using a Monte Carlo based ray-tracing algorithm. IEEE Trans. Commun. 53, 124-130 (2005). doi:10.1109/tcomm.2004.840625

16. AM Baldridge, SJ Hook, Cl Grove, G Rivera, The ASTER spectral library version 2.0. Remote Sens. Environ 113, 711-715 (2009). doi:10.1016/j.rse.2008.11.007

17. V Degli-Esposti, F Fuschini, EM Vitucci, G Falciasecca, Speed-up techniques for Ray tracing field prediction models. IEEE Trans. Antennas Propagat. 57(5), 1469-1480 (2009). doi:10.1109/TAP.2009.2016696

doi:10.1186/1687-1499-2013-7

Cite this article as: Rodríguez et al: Simulation of impulse response for indoor visible light communications using 3D CAD models. EURASIP Journal on Wireless Communications and Networking 2013 2013:7.

\section{Submit your manuscript to a SpringerOpen ${ }^{\odot}$ journal and benefit from:}

- Convenient online submission

- Rigorous peer review

- Immediate publication on acceptance

- Open access: articles freely available online

High visibility within the field

- Retaining the copyright to your article

Submit your next manuscript at $>$ springeropen.com 